Conclusion The LT-B4 level in ACS patients elevated significantly which might be positive correlated with the severity of coronary artery disease, and could promote the formation of vulnerable plaque. Plasma Leukotriene-B4 testing can improve the accuracy of detection of coronary artery plaque by 64 -slice spiral CT in patients with ACS, and have certain prediction effect of the diagnosis of the ACS and the judgements of coronary lesions. Currently, leukotriene receptor antagonist have been widely used in allergic inflammation in the respiratory system, but the clinical application in atherosclerotic disease is still being studied. Therefore, in-depth study the molecular mechanisms of 5-lipoxygenase /leukotriene pathway in the process of acute coronary syndrome may play an important role in the prediction and control of ACS and the vulnerable plaque.

\section{e0437 THE CHANGES AND SIGNIFICANCE OF COMBINED DETECTION MCP1 AND RANTES CHEMOTACTIC FACTORS IN PATIENTS WITH ACUTE CORONARY SYNDROME}

doi:10.1136/hrt.2010.208967.437

Yang Lixia, Qi Feng, Guo Ruiwei, Wang Xianmei, Guo Chuanming. Department of Cardiology Kunmin General Hospital of Chinese Plakunming

Objective To investigate implication of combination detection MCP1 and RANTES chemotactic factors in patients with ACS compare to the traditional detection of hs-CRP. And to investigate the significance of the combined detection of a variety of chemokines in early identification, risk stratification, prognosis of ACS.

Methods The 300 patients were divided into Coronary Heart Disease (CHD) group $(n=240)$ and control group $(n=60)$ according to the Coronary Angiography (CAG), and CHD group were divided into acute coronary syndrome (ACS) group $(n=180)$ and stable angina pectoris (SAP) group $(n=60)$. The severity and extent of coronary lesions was analysed by CAG and typified by means of Gensini coronary score system. Linked immunosorent assay was used to measure the concentration of MCP-1, RANTES and hs-CRP. At the same time venous blood samples were collected and total cholesterol (TC) triglyceride (TG), high density lipoprotein cholesterol (HDL-C), low density lipoprotein cholesterol (LDL-C), and red blood cells, white blood cells, platelets count, fibrinogen, and liver and kidney function were detected by automatic biochemical analyser determination.

Results Significantly increasing of MCP-1, RANTES, hs-CRP concentration, blood glucose, LDL-C levels were observed in ACS group compared to the SAP group and the control group $(p<0.05)$. And significantly decreasing of HDL-C concentration in ACS group were observed compared to the SAP group and control group. The accuracy of ACS prediction by combination detection MCP-1 and RANTES according to logistic regression equation is much better than the traditional detection of hs-CRP $(90.6 \%$ vs $82.8 \%)$.

Conclusions Combined with clinical assessment of the actual occurrence of cardiovascular disease using a variety of risk factors, we believe that coronary heart disease and acute coronary syndrome is a complex network systems regulated by multi-element, multi-factor, looking for a single factor as markers for diagnosis of coronary heart disease ACS may be limited. Combined detection of a variety of cytokines which involved in the occurrence of coronary heart disease, and through comprehensive analysis of a number of cytokines to predict cardiac events may more accurately reflect the nature of acute coronary syndrome. MCP-1, RANTES chemokine play a more specific role in monocytes /macrophages, they play a key role in the development and rupture of vulnerable plaque in coronary heart disease, especially in ACS. The effect of combination detection chemotactic factors to predict ACS is better compare to general hsCRP measurement, multi-chemotactic factors' combination detection maybe come to markers of early identification of ACS.

\section{e0438 BONE MARROW CELL THERAPY IMPROVES SYSTOLIC FUNCTION WHEREAS HAS NO EFFECT ON REMODELLING OF LEFT VENTRICULAR IN PATIENTS WITH ACUTE MYOCARDIAL INFARCTION: A META-ANALYSIS OF RANDOMISED CONTROLLED TRIALS}

doi:10.1136/hrt.2010.208967.438

${ }^{1}$ Bai Yongyi, ${ }^{2}$ Sun Lan, ${ }^{3}$ Sun Tao, ${ }^{4} Y_{e}$ Ping. ${ }^{1}$ Department Of Geriatric Cardiology, Chinese Pla General Hospital; ${ }^{2}$ National Center For Pharmaceutical Screening; ${ }^{3}$ Institute Of Materia Medica; ${ }^{4}$ Chinese Academy of Medical Sciences \& Peking Union Medical College

Objectives The objective is to perform a meta-analysis of clinical trials that investigated the effects of bone marrow cell (BMC) therapy on left ventricular (LV) function and LV remodelling in patients after acute myocardial infarction (AMI).

Background Intracoronary injection of BMCs in the acute phase of myocardial infarction has been proposed to replace cardiomyocytes lost and prevent deleterious pathological remodelling after myocardial infarction. Previously published trials have investigated the effects of cell therapy on LV function and remodelling in AMI patients. However, the sample size of these studies is small and the conclusions are inconsistent.

Methods Trials were identified in Cochrane Library, EMBASE, and PubMed databases, reviews, and reference lists of relevant papers. The weighted mean difference (WMD) was calculated for net changes in LV ejection fraction (LVEF) and LV end-diastolic volumes (LVEDV) by using random or fixed-effect models.

Results Ten randomised controlled trials (12 comparisons) with a total of 814 participants were included. In an overall pooled estimate, compared with the control group, BMCs therapy significantly improved the LVEF change from baseline to follow-up (WMD: $3.79 \%, 95 \%$ CI $2.4 \%$ to $5.7 \%, p<0.001)$. However, compared with the control group, stem cell therapy did not influence the LVEDV changes from baseline to follow-up (WMD: $-1.76 \mathrm{ml}, 95 \% \mathrm{CI}-4.61$ to $1.08 \mathrm{ml}, \mathrm{p}=0.233$ ).

Conclusion This meta-analysis suggests that cell therapy improves left ventricular contractility, whereas has no effect on LV remodelling.

\section{Q0439 RELATIONSHIP BETWEEN N-TERMINAL PRO-B-TYPE NATRIURETIC PEPTIDE AND GRACE RISK STRATIFICATION}

doi:10.1136/hrt.2010.208967.439

Han Zhao, Wenxian Liu. Capital Medical University Affiliated Beijing Anzhen Hospital

Objective To study the relationship between NT-proBNP levels obtained on admission and GRACE risk score as well as risk stratification in patients with NSTEACS (UA/NSTEMI)

Methods We enrolled 126 patients with unstable angina or Non-STsegment elevation myocardial infarction that admitted in our hospital from June of 2009 to May of 2010, 84 of the patients with UA and 42 of them with NSTEMI. Then measured their concentration of plasma NT-proBNP, cTnI, CK-MB, liver and kidney function, blood coagulation function and other Routine laboratory tests on admission. All the patients received echocardiography evaluation and 124 of them underwent angiographic examination. All the patients received risk assessment based on Clinical data, the Global Registry of Acute Coronary Events (GRACE) score which include 8 variables (age, heart rate, systolic blood pressure, serum creatinine level, Killip class at admission, presence of ST-depression, elevated cardiac biomarkers, cardiac arrest)were used to evaluate Clinical Risk. After calculate the GRACE score, the patients were stratified into three levels. Analyse the relationship between NTproBNP level and GRACE risk score in patients with NSTEACS. 
Results The lgNT-ProBNP in patients with UA and NSTEMI have positive correlation with their GRACE risk score, correlation coefficients were 0.40 and 0.52 , respectively $(p<0.05)$; the correlation coefficient of NT-proBNP level and GRACE risk score for all the patients $(n=126)$ was $0.59(p<0.05)$. After GRACE risk stratification, IgNT-ProBNP of high-risk group was the highest among the three groups $(p<0.05)$, however, the difference of GRACE score between middle-risk group and low-risk group had no statistical significance $(p>0.05)$. The lgNT-ProBNP in high-risk group was higher than non- high-risk group.

Conclusion Increased NT-proBNP level was associated with increased GRACE score in NSTEACS patients; NT-proBNP level of high-risk group increased significantly and was higher than nonhigh-risk group. NT-proBNP level in patients with NSTEACS was related to clinic risk and valuable for risk stratification in patients with NSTEACS.

\section{e0440 PREGNANCY-ASSOCIATED PLASMA PROTEIN-A POLYMORPHISMS AND THE RISKS OF ACUTE CORONARY SYNDROME}

doi:10.1136/hrt.2010.208967.440

${ }^{1}$ Caixia Tian, ${ }^{1}$ Weichao Qin, ${ }^{2}$ Weidong Zheng, Tangxin Liu. ${ }^{1}$ Renmin Hospital of Yunyang Medical College; ${ }^{2}$ Taihe Hospital of Yunyang Medical College

Background Pregnancy-associated plasma protein-A (PAPP-A) is known to be abundantly expressed in vulnerable plaques in arteriosclerotic disease. Studies have shown PAPP-A to be a sensitive biomarker of plaque instability and cardiovascular events in patients with acute coronary syndrome. This paper tried to determine the association of PAPP-A polymorphisms with acute coronary syndrome (ACS).

Methods A case-control study of 210 patients with ACS and 204 unrelated age and sex matched controls was performed. four single nucleotide polymorphisms (SNPs) of PAPP-A gene variants were detected by PCR-restriction fragment length polymorphism (PCRRFLP). The serum level of PAPP-A was measured using a newly developed sandwich ELISA technique based on 2 monoclonal antibodies.

Results Mean PAPP-A values were significantly higher in patients with acute coronary syndrome than in those with stable angina pectoris (29.7 vs $15.8 \mathrm{mIU} / \mathrm{l}, \mathrm{p}<0.01$ ). In samples drawn $<2 \mathrm{~h}$ after admission, the sensitivity of PAPP-A was superior $(93 \%)$ to that of CK-MB (60\%) and troponin T (61\%). In the patients with high-risk unstable angina pectoris, PAPP-A was related to the risk of nonfatal myocardial infarction $(p=0.02)$ but not death $(p=0.08)$. This result was consistent on multivariate analysis of the combination of mortality or nonfatal myocardial infarction (OR 2.65, 95\% CI 1.40 to 5.03). In patients with non-ST-elevation acute coronary syndrome and ST elevation myocardial infarctions, PAPP-A was related to the risk of death $(p=0.01)$. This was also true after adjustment for other univariate predictors of death (OR 2.19, 95\% CI 1.16 to 4.16). Multiple logistic regression analysis with risk factors such as age, male sex, smoking, hypertension, diabetes mellitus, and dyslipidemia revealed the PAPP-A IVS6+95 C allele (dbSNP: rs13290387) to be associated with an increased risk of ACS (OR, 2.44; 95\% CI 1.21 to $3.98 ; \mathrm{p}=0.018)$. The IVS6+95 (G/C) polymorphism in the PAPP-A gene has been reported 102 cases $(48.6 \%)$ were GG and 80 cases (38.1\%) were GC and 28 cases $(13.3 \%)$ were CC for the ACS group; the respective figures were 116 $(56.9 \%)$ and $70(34.3 \%)$ and $18(8.8 \%)$ in the controls. Patients carrying the $\mathrm{C}$ allele had a tendency to increased risk of ACS.

Conclusions In the early stages of non-ST-elevation acute coronary syndrome and ST elevation myocardial infarctions, PAPP-A seems to be a more sensitive marker of myocardial infarction than CK-MB and troponin T. PAPP-A seems to be valuable in predicting the outcomes of patients admitted with high-risk NSTE-ACS or STEMI PAPP-A IVS6+95 C allele is an independent risk factor for ACS even after adjustment for traditional risk factors.

\section{E0441 SAFETY OF AGGRESSIVE ANTI-THROMBOTIC THERAPY IN ELDERLY PATIENTS WITH PERSISTENT ST ELEVATED MYOCARDIAL INFARCTION UNDERWENT PRIMARY PERCUTANEOUS CORONARY INTERVENTION - A SINGLE CENTER AND SINGLE OPERATOR EXPERIENCE}

doi:10.1136/hrt.2010.208967.441

Li Yi, Hu Chengheng, Du Zhimin, Ma Hong, He Jiangui, Liu Jun. First Affiliated Hospital of Sun Yat-sen University

Objectives To evaluated the safety and efficacy of individualised anti-thrombotic therapy in elderly patients with ST-elevated myocardial infarction underwent primary PCI based on age groups. Methods Study population Between Jan. 2007 and Dec. 2008, patients with ST-elevated myocardial infarction eligible for primary PCI was assigned into 3 groups based on their ages: non-elderly group (CON, $\leq 65)$, elderly 1(ELD1, 65<age $\leq 75)$, and elderly 2 (ELD2, >75). These patients received individualised anti-thrombotic therapy based on their age group. Non-elderly patients received $300 \mathrm{mg}$ aspirin and $600 \mathrm{mg}$ clopidogrel loading dose at the emergency department and $10 \mu \mathrm{g} / \mathrm{kg}$ tirofiban loading dose were given intravenous or intra-coronary prior to intervention and followed by $0.15 \mu \mathrm{g} / \mathrm{kg} \cdot \mathrm{min}$ infusion for $36 \mathrm{~h}$. Elderly patients received $300 \mathrm{mg}$ aspirin and $300 \mathrm{mg}$ clopidogrel loading dose at the emergency department and tirofiban was given based on the thrombus burden in the culprit vessel. Clinical and angiographic parameters bleeding complications, syntax score, TIMI and CTFC coronary flow, TMP myocardial perfusion grade, in-hospital and long-term MACE, including cardiogenic death, non-fatal re-infarction, target vessel revascularization, re-hospitalisation.

Results Between Jan. 2007 and Dec. 2008, 124 patients with STelevated myocardial infarction eligible for primary PCI were enrolled. There were 48 patients in control group, 46 patients in ELD1 group, and 30 patients in ELD2 group. Patients in ELD1 group and ELD2 group had more co-morbidity factors. The complexity of coronary lesions was similar in three groups, the SYNTAX score in three groups were 17.7 $\pm 7.3,17.0 \pm 7.7$ and $16.8 \pm 6.1(p=0.829))$. The immediate angiographic outcome was also similar in three groups. The CTFC of infarction-related artery in three group were $31.4 \pm 14.1,33.3 \pm 16.9$ and $32.5 \pm 13.8(\mathrm{p}=0.279))$. TMP-3 perfusion were achieved in $79.2 \%, 71.2 \%$ and $80 \%$ patients in 3 groups. TIMI3 flow were achieved in $87.5 \%, 86.9 \%$ and $86.6 \%$ patients in 3 groups. There were no fatal bleeding and TIMI major bleeding in both groups. There was a trend of increased TIMI minor bleeding risk in ELD2 group patients.

Conclusion Our single-center and single-operator experience indicate that individualised aggressive anti-thrombotic therapy for elderly patients with ST-elevated myocardial infarction underwent primary PCI could improve myocardial perfusion and coronary flow. Individualised aggressive anti-thrombotic therapy for elderly patients with ST-elevated myocardial infarction underwent primary PCI did not increase the bleeding risk.

\section{e0442 EFFECT OF ASPIRIN AND CILOSTAZOL ON INFLAMMATORY CYTOKINES IN PATIENTS WITH ACUTE CORONARY SYNDROME}

doi:10.1136/hrt.2010.208967.442

${ }^{1}$ Hao Tang, ${ }^{2}$ Jingguo Wu, ${ }^{2}$ Qing Yang, ${ }^{3}$ Zhongfu Ma. ${ }^{1}$ Department of General Internal Medcine of The First Affiliated Hospital of Sun Yat-sen University, Guangzhou; ${ }^{2}$ Department of General Internal Medcine of The First Affiliated Hospital of Sun Yat-sen 